\title{
Multiband Maximum Likelihood Signal Detection Based on Compressive Measurements
}

\author{
Jonathan Verlant-Chenet Jonathan Bodart André Bourdoux* \\ Jean-Michel Dricot Philippe De Doncker François Horlin \\ Université Libre de Bruxelles - OPERA Dpt., Avenue F.D. Roosevelt 50, B-1050 Brussels, Belgium \\ *Interuniversity Microelectronics Centre, Kapeldreef 75, B-3001 Leuven, Belgium \\ jverlant@ulb.ac.be
}

\begin{abstract}
Cognitive radios impose challenges on the design of efficient signal detectors, including wide bandwidth sensing and large dynamic range support. The recently considered compressed sensing theory helps in relaxing the constraints on the design of the analog front-end. The maximum likelihood method introduced here is computationally simple since it does not require a signal reconstruction, unlike most methods introduced in the current literature. Moreover, the metric is optimum, works for any modulation scheme and is independent of the emitted signal knowledge and the number of occupied bands. The results are supported with Matlab simulations, a statistical study is performed and the probabilities of misdetection and false alarm are plotted for different scenarios, proving the efficiency of the estimator in a range of plausible SNRs and subsampling factors.
\end{abstract}

\section{INTRODUCTION}

Many studies have shown that static frequency allocation for wireless communication systems is responsible for the inefficient use of the spectrum [1]: communication systems are not transmitting continuously, which causes gaps in the frequency spectrum that vary over time. Cognitive radios (CR) try to make use of these gaps by first detecting and then using them for their own transmission (spectrum sensing and communication steps). This opportunistic behavior categorizes $\mathrm{CR}$ as secondary users of a given frequency band, by contrast to the systems that were permanently assigned this band (primary users) [2]. For the CR concept to be viable, it is required that it does not interfere with the primary user services. As a consequence, the CR system must be able to detect primary user signals under rigorous time and reliability constraints. Efforts are being made to design efficient signal detectors that can cope with the new challenges imposed by the CR systems [3]. The detectors should first be capable of scanning a wide bandwidth with a large dynamic range to detect primary user signals that occupy sparse frequency bands and originate from a large range of distances from the detector. This makes the implementation of the analog front-end and digital processor difficult [4].

Recently the theory of compressed sampling has received an increasing attention as it may help in relaxing the constraints on the design of the CR system. It is based on the fact that a frequency sparse signal may be sampled at a rate significantly lower than the Nyquist rate without loosing information. This may potentially facilitate the implementation of the analog front-end (lower bandwidth filters, lower sampling rate analogto-digital (A/D) converters) and digital processor (lower rate signal processing).

Landau has computed a lower bound on the sampling density required to enable the perfect reconstruction of a continuous time signal from the samples [5]. This bound applies to an arbitrary sampling scheme, uniform or not, and is not necessarily achievable. The theory of compressed sampling (CS) investigates how the Landaus lower bound can be approached in practice [6]. It studies the conditions under which a signal having a sparse representation in one basis can be recovered from a small number of projections onto a second basis that is incoherent with the first. It demonstrates that the perfect reconstruction of multi-band signals is for example possible from non-uniformly spaced samples taken at sub-Nyquist average rate approaching the Landaus lower-bound. In this case, the signal representation basis is the Fourier basis (the signal is sparse in the frequency domain) while the measurement basis is the canonical basis (the signal is sampled non-uniformly in the time domain). The Fourier and canonical bases are maximally incoherent. Interestingly, random waveforms with independent and identically distributed (i.i.d.) entries, e.g. Gaussian or binary entries, exhibit a very low coherence with any fixed representation basis with a high probability. Therefore they provide a useful universal measurement basis and generic hardware can be built for the acquisition of a variety of signal classes [7]. In the CS framework, signal recovery is achieved through expensive non-linear optimization or iterative algorithms. The signal reconstruction may be expressed as a problem of $\ell_{1}$-norm minimization subject to a linear equality constraint and typically solved with linear programming algorithms. For example, [8] proposes a specialized interior-point method for solving large sparse problems such as the signal recovery. However the $\ell_{1}$-minimization is not the only way to recover sparse solutions. Other methods such as the orthogonal matching pursuit algorithm have been proposed [9]. Signal reconstruction is not necessary in many signal processing applications. Very often, the designer is only interested in solving an inference problem (extracting a well-defined information from the measurements) or in filtering out information of no interest before further processing. Based on the CS theory, the designer could attempt to recover the full signal from the compressive measurements and then solve the inference or filtering problem using traditional digital signal processing (DSP). However this approach is suboptimal in terms of both accuracy and digital complexity [10]. The best solution is to solve the signal processing problem in the compressive domain without first resorting to full scale signal reconstruction. Davenport et al. tackle the problems of the detection of a known signal in noise, the classification of signals, the estimation of a linear function of the data, and the filtering out of the interference [11].

In this paper, the approach is to directly detect the presence of the primary user signals of expected modulation parameters in the compressive domain without having first to resort to the reconstruction of the wideband signal spectrum. We will ex- 
tend our former contributions [12], [13] that both consider a wideband channel composed of a large number of subchannels that are independently scanned for potential reuse by the secondary network. While [12] assumes that the subchannels are composed of unmodulated carrier frequencies, [13] extends the scheme to linearly modulated signals and introduces an optimum detector valid for scenarios with only one occupied band. The proposed method can be viewed as an extension of the signal classification investigated in [11], [13] to linearly modulated signals with unknown symbols and unknown number of occupied bands.

The paper is organized as follows. We first introduce the system model in Section II. Then, we compute the likelihood function in Section III. An analytical study of this function is performed and the results are supported with Matlab simulations in Section IV.

\section{SYSTEM MODEL}

\section{A. Temporal model}

Fig. 1 illustrates the overall system when linearly modulated communication signals are present at $\mathrm{K}$ center frequencies $f_{k}$.

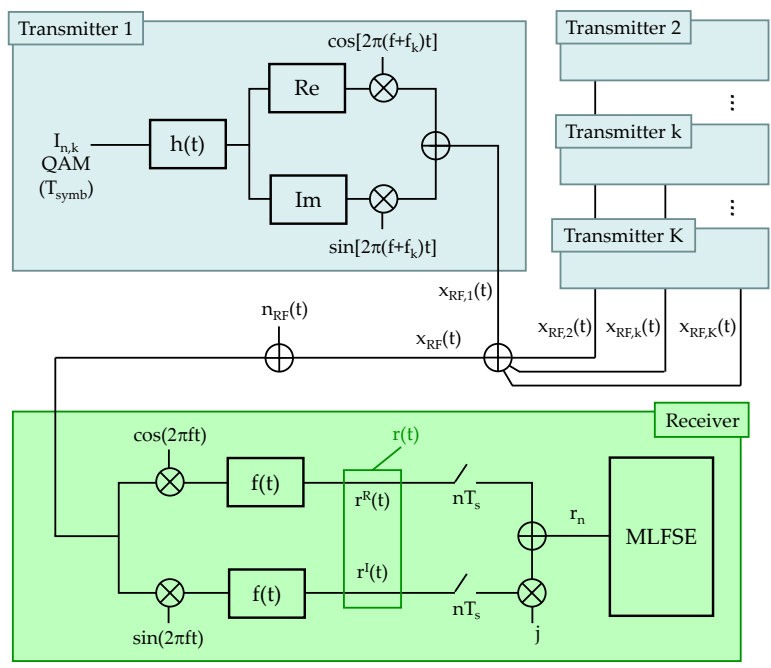

Fig. 1. Bandpass architecture with K occupied bands.

At each transmitter, independent QAM modulated symbols $I_{n, k}$ of variance $\sigma_{I}^{2}$ are transmitted at the rate $1 / T_{\text {symb. The }}$ symbols are supposed independent and identically distributed (i.i.d.), with null mean and no correlation between real and imaginary parts. Note that the detector metric computed in Section III will be independent of the transmitted symbols (and therefore also of the modulation scheme). The sequence is lowpass filtered by $h(t)$ in order to remove the out-of-band components (typically a normalized Square Root Raised Cosine filter is used), and then modulated on frequency $f_{k}$. The resulting baseband signal, for all transmitters, is given by:

$$
x(t)=\sum_{k=1}^{K} \sum_{n} I_{n, k} h\left(t-n T_{\mathrm{symb}}\right) e^{j 2 \pi f_{k} t}
$$

The variance of a signal emitted in one band $x_{k}(t)$ is equal to:

$$
\sigma_{x_{k}}^{2}=\sigma_{I}^{2} \frac{1}{T_{\text {symb }}} \int_{-\infty}^{\infty}|h(t)|^{2} d t
$$

where $M$ is the number of bands in the total available bandwith. The radio-frequency signal $x_{\mathrm{RF}}(t)$ is obtained after upconversion to the carrier frequency $f$ and transmitted through the channel, where additive white Gaussian noise $w_{\mathrm{RF}}(t)$, of one-sided power spectral density (PSD) equal to $N_{0}$, corrupts the received signal.

At the receiver, the RF signal is down-converted to the baseband domain for complex operation and low-pass filtered by $f(t)$ in order to limit the observation to the desired bandwidth $B_{f}=\frac{1}{T_{s}}$, where $\frac{1}{T_{s}}$ is the Nyquist sampling rate. As we assume an additive white gaussian noise channel with no multipath, the baseband received signal $r(t)$ can be expressed as:

$$
r(t)=x(t)+w(t)
$$

where $w(t)$ is the baseband equivalent noise of variance $\sigma_{w}^{2}=2 N_{0} \frac{1}{T_{s}}$. Thus, the noise for one single band of the system is $\frac{1}{M} \sigma_{w}^{2}$. We define the signal-to-noise ratio as the power of one signal divided by the power of the noise in a single band:

$$
S N R=\frac{\sigma_{I}^{2}}{\frac{1}{M} \sigma_{w}^{2}}
$$

After classical sampling, the received sequence $r_{m}$ is used for the spectrum sensing (here called MLFSE for Maximum Likelihood Frequency Set Estimator, see Section III).

\section{B. Subsampling and matricial model}

In our scenario, the total available bandwith is subdivided in $M$ bands, and the number $K$ of occupied bands is unknown. The detector will have two tasks : evaluate $K$ and choose which of these $\mathrm{K}$ bands are occupied. For instance, 3 users are occupying 3 of the ten $10 \mathrm{MHz}$ channels available in a range of $100 \mathrm{MHz}$. This scenario relies on subsampling at rates that do not respect the Nyquist criterion (i.e. inferior to $100 \mathrm{MHz}$ ), such as $40 \mathrm{MHz}$ (subsampling by a factor 2.5).

The received signal is subsampled at $\mathrm{P}$ random instants $t_{p}$, with a mean rate $1 / T_{\text {sub }}$, smaller then the Nyquist rate. The obtained received sequence is:

$$
r_{p}=r\left(t_{p}\right)=\sum_{k=1}^{K} \sum_{n} I_{n, k} h\left(t_{p}-n T_{\mathrm{symb}}\right) e^{j 2 \pi f_{k} t_{p}}+w\left(t_{p}\right)
$$

This sequence can be expressed as a matricial product:

$$
\underline{r}=\underline{\underline{S}} \cdot\left(\sum_{k=1}^{K} \underline{\underline{\Phi_{k}}} \cdot \underline{\underline{H_{k}}} \cdot \underline{I_{k}}+\underline{w}\right)
$$

where $I_{k}$ is a vector containing the N QAM symbols emitted by the $k^{t h}$ user that are kept in the window of observation and $\underline{r}$ is a vector containing $\mathrm{P}$ received samples. The $\mathrm{N}$ symbols are multiplied by a convolution matrix $\underline{\underline{H_{k}}}$ of size $D \times N$ (representing the convolution with the Square Root Raised Cosine filter) 
and a frequency shift diagonal matrix $\underline{\underline{\Phi_{k}}}$ containing exponential factors, such as:

$$
\Phi_{d d^{\prime}, k}= \begin{cases}\exp \left(j 2 \pi f_{k} t_{d}\right) & \text { if } d=d^{\prime} \\ 0 & \text { else }\end{cases}
$$

$\underline{S}$ is the subsampling rectangular matrix of dimensions $P \times D$ containing only one non-null element on each line corresponding to the random times of sampling.

Let us define a matrix $\underline{\underline{\Phi}}$ as a horizontal juxtaposition of all the $\underline{\underline{\Phi_{k}}}$, that is:

$$
\underline{\underline{\Phi}}=\left(\underline{\underline{\Phi_{1}}} \underline{\underline{\Phi_{2}}} \cdots \underline{\underline{\Phi_{K}}}\right)
$$

a matrix $\underline{\underline{H}}$ as a diagonal juxtaposition of all the $\underline{\underline{H_{k}}}$, that is:

$$
\underline{\underline{H}}=\left(\begin{array}{cccc}
\underline{\underline{H_{1}}} & 0 & 0 & 0 \\
0 & \underline{\underline{H_{2}}} & 0 & 0 \\
0 & 0 & \ddots & 0 \\
0 & 0 & 0 & \underline{\underline{H_{K}}}
\end{array}\right),
$$

and a vector $\underline{I}$ as a vertical juxtaposition of all the $\underline{I_{k}}$, that is:

$$
\underline{I}=\left(\begin{array}{c}
\frac{I_{1}}{I_{2}} \\
\vdots \\
\underline{I_{K}}
\end{array}\right) .
$$

With these three definitions, the expression (4) becomes:

$$
\underline{r}=\underline{\underline{S}} \cdot \underline{\underline{\Phi}} \cdot \underline{\underline{H}} \cdot \underline{I}+\underline{\underline{S}} \cdot \underline{w}
$$

\section{Maximum Likelihood Frequency Set Estimator (MLFSE)}

Since Maximum Likelihood estimation is used to estimate parameters of a statistical model, we will use it to find the central frequencies of the bands in use. The criterion is to maximize the probability density function of the signal while knowing the frequency set $\phi=\left\{f_{k}\right\}_{k \in[1 ; K]}[14]$ :

$$
\widehat{\phi}=\arg \max _{\phi} p(\underline{r} \mid \phi) \text {. }
$$

Therefore:

$$
\begin{aligned}
\widehat{\phi} & =\arg \max _{\phi} \int_{\underline{I}} p(\underline{r} \mid \phi, \underline{I}) p(\underline{I}) \\
& =\arg \max _{f} \mathbb{E}_{\underline{I}}[p(\underline{r} \mid \phi, \underline{I})]
\end{aligned}
$$

where $\mathbb{E}_{\underline{I}}$ denotes the expectation with respect to the symbols $\underline{I}$.

\section{A. PDF computation}

The purpose of this subsection is to compute the PDF $p(\underline{r} \mid \phi, \underline{I})$. In the expression (9) of the received signal, the first term is deterministic as $\underline{I}$ is supposed to be known; only the second term is random and has a Gaussian density of probability by definition of the AWGN. Thus, the PDF of $\underline{r}$ is the same as a Gaussian random variable with mean $\underline{\underline{S}} \cdot \underline{\underline{\Phi}} \cdot \underline{\underline{H}} \cdot \underline{I}$ and variance $\sigma_{w}^{2} \underline{\underline{I}}_{P}$ (where $\underline{\underline{I}}_{P}$ denotes the identity matrix of size P).

We obtain:

$$
\begin{aligned}
p(\underline{r} \mid \phi, \underline{I}) & =C \cdot \exp \left(-\frac{1}{2 \sigma_{w}^{2}}|\underline{r}-\underline{S} \cdot \underline{\underline{\Phi}} \cdot \underline{\underline{H}} \cdot \underline{I}|^{2}\right) \\
& =C \cdot \exp \left(-\frac{1}{2 \sigma_{w}^{2}}\left(\underline{r}^{H} \cdot \underline{r}-2 \Re\left[\underline{y}^{H} \cdot \underline{I}\right]+\Lambda\right)\right)
\end{aligned}
$$

where

- $C=\left(2 \pi \sigma_{n}^{2}\right)^{\frac{N}{2}}$ is independent of $\underline{I}$ and $\phi$

- $\underline{r}^{H} \cdot \underline{r}$ is independent of $\underline{I}$ and $\phi$ as $\underline{r}$ is measured at the receiver

- $\underline{y}=\underline{\underline{H}}^{H} \cdot \underline{\Phi}^{H} \cdot \underline{\underline{S}}^{H} \cdot \underline{r}$ is a matched filter operation on $\underline{r}$. It does not depend on $\underline{I}$ but will affect the maximization with respect to $\phi$ (the dependency being in the $\underline{\underline{\Phi}}$ matrix) in (11).

- $\Lambda=\underline{I}^{H} \cdot \underline{H}^{H} \cdot \underline{\Phi}^{H} \cdot \underline{S}^{H} \cdot \underline{S} \cdot \underline{\Phi} \cdot \underline{\underline{H}} \cdot \underline{I}$. We know that $\underline{\underline{H}}^{H} \cdot \underline{\underline{\Phi}}^{H} \cdot \underline{\underline{\Phi}} \cdot \underline{\underline{H}}=\underline{\underline{I_{N}}}$ (where $\underline{\underline{I_{N}}}$ is the size-N identity matrix) and we use the approximation $\underline{\underline{H}}^{H} \cdot \underline{\underline{\Phi}}^{H} \cdot \underline{\underline{S}}^{H} \cdot \underline{\underline{S}} \cdot \underline{\underline{\Phi}} \cdot \underline{\underline{H}} \approx c \underline{\underline{I_{N}}}$ where $c=\frac{T_{\text {symb }}}{T_{s u b}}$ is the subsampling factor. Thus, $\Lambda=c \underline{I}^{H} \underline{I}$ is independent of $\phi$, and is (approximately) equal to $c . N . K$ in the case of PSK (QAM) symbols.

The PDF (12) can be simplified as:

$$
p(\underline{r} \mid \phi, \underline{I})=C^{\prime} \cdot \exp \left(-\frac{1}{2 \sigma_{w}^{2}}\left(\underline{y}^{H} \underline{I}+\underline{I}^{H} \underline{y}\right)\right)
$$

where $C^{\prime}=C \cdot \exp \left(-\frac{1}{2 \sigma_{w}^{2}}\left(\underline{r}^{H} \cdot \underline{r}+c N K\right)\right)$ is a factor that does not affect the expectation nor the maximization with respect to $\phi$.

For low $S N R$, the factor $-\frac{1}{2 \sigma_{w}^{2}}$ is small and the exponential can be expanded as a Maclaurin serie:

$$
\exp (\lambda) \approx \sum_{\alpha=0}^{\infty} \frac{\lambda^{\alpha}}{\alpha !}
$$

As cognitive radios often deal with low $S N R s$, this expansion will be used in the next section.

\section{B. Likelihood function computation}

The likelihood function has the form:

$$
\begin{aligned}
\max _{\phi} \mathbb{E}_{\underline{I}}[p(\underline{r} \mid \phi, \underline{I})] & =\max _{\phi} \mathbb{E}_{\underline{I}}\left[C^{\prime} \exp (\lambda)\right] \\
& \approx \max _{\phi} \mathbb{E}_{\underline{I}}\left[C^{\prime} \sum_{\alpha=0}^{\infty} \frac{\lambda^{\alpha}}{\alpha !}\right] \\
& \approx \max _{\phi} C^{\prime} \sum_{\alpha=0}^{\infty} \frac{1}{\alpha !} \mathbb{E}_{\underline{I}}\left[\lambda^{\alpha}\right]
\end{aligned}
$$

where $\lambda=-\frac{1}{2 \sigma_{w}^{2}}\left(\underline{y}^{H} \underline{I}+\underline{I}^{H} \underline{y}\right)$

To compute the expectation, we use the binomial theorem that states:

$$
(x+y)^{n}=\sum_{k=0}^{n}\left(\begin{array}{l}
n \\
k
\end{array}\right) x^{n-k} y^{k}
$$


Thus:

$$
\begin{aligned}
\mathbb{E}_{\underline{I}}\left[\lambda^{\alpha}\right] & =\left(-\frac{1}{2 \sigma_{w}^{2}}\right)^{\alpha} \mathbb{E}_{\underline{I}}\left[\left(\underline{y}^{H} \underline{I}+\underline{I}^{H} \underline{y}\right)^{\alpha}\right] \\
& =\left(-\frac{1}{2 \sigma_{w}^{2}}\right)^{\alpha} \sum_{k=0}^{\alpha}\left(\begin{array}{l}
\alpha \\
k
\end{array}\right) \mathbb{E}_{\underline{I}}\left[\left(\underline{y}^{H} \underline{I}\right)^{\alpha-k}\left(\underline{I}^{H} \underline{y}\right)^{k}\right]
\end{aligned}
$$

Only the terms where $\left(y^{H} \underline{I}\right)$ and $\left(\underline{I}^{H} y\right)$ have the same power are non-null because symbols are i.i.d. by hypothesis and

$$
\mathbb{E}_{\underline{I}}\left[\left(I_{n}\right)^{\gamma}\right]=0 \quad \forall \gamma \in \mathbb{N} .
$$

That is when $k=\frac{\alpha}{2}$. Thus, the odd orders of the approximation disappear, and we have:

$$
\mathbb{E}_{\underline{I}}\left[\lambda^{2 \beta}\right]=\left(\frac{1}{2 \sigma_{w}^{2}}\right)^{2 \beta}\left(\begin{array}{c}
2 \beta \\
\beta
\end{array}\right) \mathbb{E}_{\underline{I}}\left[\left(\underline{y}^{H} \underline{I}\right)^{\beta}\left(\underline{I}^{H} \underline{y}\right)^{\beta}\right]
$$

with $\beta=\frac{\alpha}{2}$.

The likelihood function becomes:

$$
\max _{\phi} \mathbb{E}_{\underline{I}}[p(\underline{r} \mid \phi, \underline{I})] \approx \max _{\phi} \sum_{\beta=1}^{\infty} \frac{1}{\left(\beta ! 2^{\beta} \sigma_{w}^{2 \beta}\right)^{2}} G_{\beta}(\phi)
$$

where

$$
G_{\beta}(\phi)=\mathbb{E}_{\underline{I}}\left[\left(\underline{y}^{H} \underline{I}\right)^{\beta}\left(\underline{I}^{H} \underline{y}\right)^{\beta}\right]
$$

For a plausible range of SNRs, it is possible to show that terms in the likelihood function for $\beta>3$ are negligible. Let us compute the $G_{\beta}(\phi)$ function for $\beta \leq 3$.

When $\beta=1$,

$$
\begin{aligned}
G_{1}(\phi) & =\mathbb{E}_{\underline{I}}\left[\sum_{n_{1}=1}^{K . N} \sum_{n_{2}=1}^{K . N} I_{n_{1}} I_{n_{2}}^{*} y_{n_{1}}^{*} y_{n_{2}}\right] \\
& =\sigma_{I}^{2} \sum_{n}^{K . N} y_{n}^{*} y_{n}=\sigma_{I}^{2} \underline{y}^{H} \underline{y}
\end{aligned}
$$

because $\mathbb{E}_{\underline{I}}\left[I_{n_{1}} I_{n_{2}}^{*}\right]$ is different of 0 only when $n_{1}=n_{2}$. When $\beta=2$,

$$
\begin{aligned}
G_{2}(\phi) & =\mathbb{E}_{\underline{I}}\left[\sum_{n_{1}=1}^{K . N} \sum_{n_{2}=1}^{K . N} \sum_{n_{3}=1}^{K . N} \sum_{n_{4}=1}^{K . N} I_{n_{1}} \ldots I_{n_{4}}^{*} y_{n_{1}}^{*} \ldots y_{n_{4}}\right] \\
& =2 \sigma_{I}^{4}\left(\underline{y}^{H} \underline{y}\right)^{2}-\sigma_{I}^{4} \sum_{n}^{K . N}\left|y_{n}\right|^{4} .
\end{aligned}
$$

The first term in (22) comes from the fact that $\mathbb{E}_{\underline{I}}\left[I_{n_{1}} \ldots I_{n_{4}}^{*}\right]$ is different of 0 only when $\left\{\begin{array}{l}n_{1}=n_{3} \\ n_{2}=n_{4}\end{array}\right.$ and $\left\{\begin{array}{l}n_{4}=n_{3} \\ n_{2}=n_{3}\end{array}\right.$, and the second term is there to correct the fact that we counted two times the case where $n_{1}=n_{2}=n_{3}=n_{4}$.

Using the set theory and the inclusion-exclusion principle, it is possible to show that, when $\beta=3$,

$$
\begin{aligned}
G_{3}(\phi) & =\mathbb{E}_{\underline{I}}\left[\sum_{n_{1}=1}^{K . N} \ldots \sum_{n_{6}=1}^{K . N} I_{n_{1}} \ldots I_{n_{6}}^{*} y_{n_{1}}^{*} \ldots y_{n_{6}}\right] \\
& \left.=6 \sigma_{I}^{6} \underline{y}^{H} \underline{y}\right)^{3} \\
& -9 \sigma_{I}^{6} \sum_{n_{1}}^{K . N}\left|y_{n_{1}}\right|^{4} \sum_{n_{2}}^{K . N}\left|y_{n_{2}}\right|^{2} \\
& +4 \sigma_{I}^{6} \sum_{n}^{K . N}\left|y_{n}\right|^{6}
\end{aligned}
$$

The likelihood function becomes:

$$
\max _{\phi} \mathbb{E}_{\underline{I}}[p(\underline{r} \mid \phi, \underline{I})] \approx \max _{\phi} \sum_{\beta=1}^{3} \frac{1}{\left(\beta ! 2^{\beta} \sigma_{w}^{2 \beta}\right)^{2}} G_{\beta}(\phi)
$$

This function has a very simple form which has only one dependency on $\phi$ (through the $\underline{y}$ vectors). Moreover, it can be computed as a simple dyadic product.

\section{Simulations}

To support previous analytical results with Matlab simulations, we implemented the MLFSE for the mentioned scenario. For this simulation, the estimator is computing $\mathbb{E}_{\underline{I}}[p(\underline{r} \mid \phi, \underline{I})]$ based on a $3 \mu s$ observation window. This likelihood function is computed for each set combining frequency carriers ranging from $-45 \mathrm{MHz}$ to $45 \mathrm{MHz}$ (i.e. $100 \mathrm{MHz}$ baseband total bandwidth) every $10 \mathrm{MHz}$. There are two different scenarios: one where the number of users is known $(K=2)$ and one when it is not. The latter is thus more complex as we have to compute our metric for a larger set of frequencies $\left\{\phi_{k}\right\}_{1 \leq k \leq 5}$. We assume that $K \leq 5$ because the subsampling factor is limited by the number of users, and the probability of having more than 5 users is below $10^{-5}[2]$.

Let us now use this context to perform a statistical study of our frequency set estimator. For that, we execute 5000 realizations of the estimation and compute the probabilities of misdetection $p_{M D}$ and false alarm $p_{F A}$. Those probabilities depend on the $S N R$ defined in (3) and the subsampling factor $c$ (ratio between the Nyquist minimum sampling frequency and the subsampling mean rate), which are the two parameters of our simulations.

Fig. 2 is the simulation result for the case where $K$ is known to be equal to 2 . It shows the probability of misdetection $p_{M D}$ (here equal to $p_{F A}$ ) for three different mean sampling rates : 50, 40, and $20 \mathrm{MHz}$. It quickly rises for SNRs below a certain value. Above this value, the probability tends to zero and our estimator is reliable. As expected, when the subsampling factor $c$ increases (i.e. when the mean sampling rate decreases), the estimator performance decreases and the curves are shifted to the right.

Fig. 3 further shows the performance of the estimator when the number $K$ of users is unknown (compared to the case where 


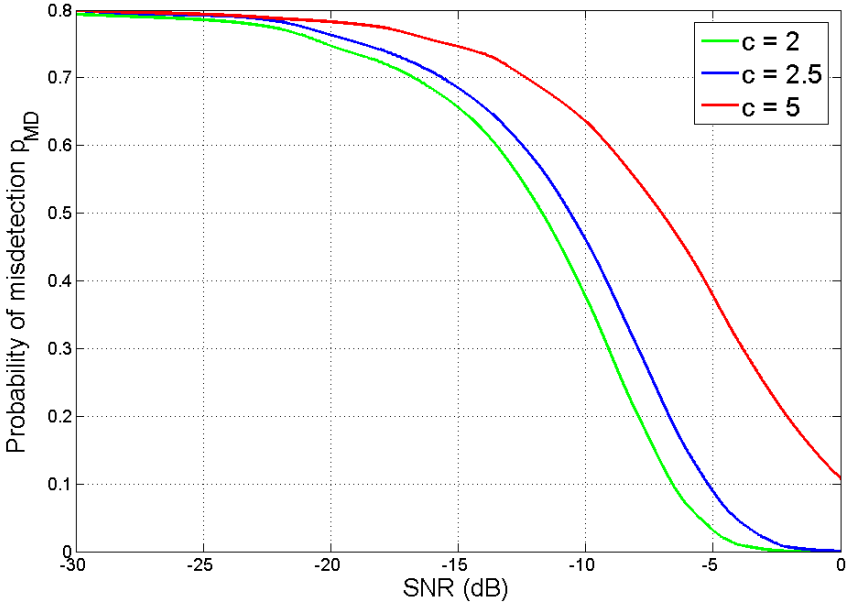

Fig. 2. Probability of misdetection $p_{M D}$ for a known number of users $K=2$ in the case of subsampling factors $2,2.5$ and 5 (respectively corresponding to mean sampling rates 50, 40 and $20 \mathrm{MHz}$ ).

$K$ is known). As expected, the computing time was longer though the performance is comparable to those for a known $K$.

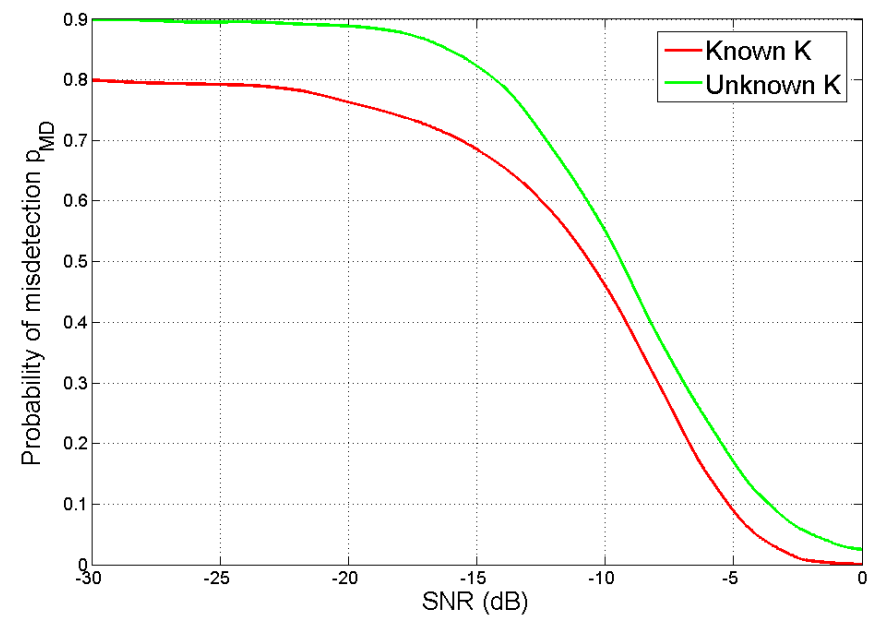

Fig. 3. Probability of misdetection $p_{M D}$ for known and unknown number $K$ of users in the case of subsampling factor 2.5 (corresponding to a mean sampling rate of $40 \mathrm{MHz}$ ).

\section{CONCLUSION}

This paper introduces a new frequency set estimator combining maximum likelihood method and subsampling. The computed metric is optimum, works for any modulation scheme and is independent of the emitted signal knowledge, which is useful for cognitive radio scenarios. As mentioned, it extends our previous works in [12] (as it supports modulated signals), in [13] (as it supports any number of occupied bands) and it extends the works of [11] as it is independant of the transmitter symbols.

A statistical study is performed and the probabilities of misdetection and false alarm are computed for varying SNR and subsampling factor.

As expected, those probabilities increase when the mean sampling frequency or the SNR decreases. The simulations prove the efficiency of the estimator in a range of plausible SNRs and subsampling factors, and also show the limits of it. Thus, for a given probability of false alarm or misdetection, it is possible to estimate a the subsampling factors that may satisfy a range of SNRs. One big advantage of this new frequency set estimator is that it metric is independant of the number of users, which is a further step towards a blind cognitive radios scenario.

Further works on this topic will take in account synchronization issues and multipath channel scenarios.

\section{REFERENCES}

[1] F.C. Commission, "Fcc-03-322: Facilitating opportunities for flexible, efficient, and reliable spectrum use employing cognitive radio technologies," 2003.

[2] I. F. Akyildiz, W. Y. Lee, M. C. Vuran, and S. Mohanty, "Next generation / dynamic spectrum access / cognitive radio wireless networks: A survey," Computer Networks Journal (Elsevier), vol. 50, pp. 2127 2159, 2006.

[3] A. Sahai and D. Cabric, "Spectrum sensing: Fundamental limits and practical challenges," in IEEE Proceedings of DySPAN, 2005.

[4] D. Cabric and R. W. Brodersen, "Physical layer design issues unique to cognitive radio systems," in IEEE Proceedings of ISPIMRC, 2005.

[5] H. J. Landau, "Necessary density functions for sampling and interpolation of certain entire functions," Acta Mathematica, vol. 117, pp. 37 - 52, 1967.

[6] D. L. Donoho, "Compressed sensing," IEEE Transactions on Information Theory, vol. 52, no. 4, pp. $1289-1306,2006$.

[7] E. J. Candes and T. Tao, "Near-optimal signal recovery from random projections," IEEE Transactions on Information Theory, vol. 52, no. 12, pp. $5406-5425,2006$.

[8] S. J. Kim, K. Koh, M. Lustig, S. Boyd, and D. Gorinevsky, "An interiorpoint method for large-scale 11-regularized least squares," IEEE Journal on Selected Areas in Communications, vol. 1, no. 4, pp. 606 - 617, 2007.

[9] J. A. Tropp and A. C. Gilbert, "Signal recovery from partial information via orthogonal matching pursuit," IEEE Transactions on Information Theory, vol. 53, no. 12, pp. 4655 - 4666, 2007.

[10] M. A. Davenport, M. Wakin, and R. Baraniuk, "Detection and estimation with compressive measurements," in Technical report, University of Rice, 2006.

[11] M. A. Davenport, P. T. Boufounos, M. B. Wakin, and R. G. Baraniuk, "Signal processing with compressive measurements," IEEE Journal on Selected Topics in Signal Processing, vol. 4, no. 2, pp. 445 - 460, 2010.

[12] A. Bourdoux, S. Polin, A. Dejonghe, and L. Van der Perre, "Tone detection of non-uniformly undersampled signals with frequency excision," in IEEE Proceedings of ICASSP, 2011.

[13] J. Verlant-Chenet, A. Bourdoux, J. M. Dricot, P. De Doncker, and F. Horlin, "Wideband spectrum sensing for cognitive radios using optimum detector with no reconstruction," in IEEE Proceedings of ICNC, 2012.

[14] H. Meyr, M. Moeneclaey, and S. Fechtel, Digital Communication Receivers: Synchronization, Channel Estimation, and Signal Processing, John Wiley and Sons, Inc., 1997. 